Revista Electrónica Teoría de la Educación.

Educación y Cultura en la Sociedad de la Información.

http://www.usal.es/teoriaeducacion

Vol. 9. No 2. Mayo 2008

\title{
CAPACITACIONES AUTÓNOMAS INDÍGENAS - RESPUESTA A LA POLÍTICA ASIMILADORA NACIONAL. EL EJEMPLO DE LOS WIXARITAR DE MÉXICO.
}

Resumen. Las condiciones de vida de los pueblos indígenas en México están terminadas por la política asimiladora del gobierno mexicano, entre otros. Bajo el llamado Indigenismo, se lleva acabo programas oficiales para la asimilación y aculturalización de los pueblos indígenas. Rechazando esa negación de autodeterminación indígena, los indígenas mexicanos reivindican el reconocimiento y la implementación de sus derechos y ponen esfuerzo en llevar a la práctica formas locales de autonomía. En el debate por la autonomía indígena, el sector de la educación representa punto nodal de gran importancia: se reclaman influencia sobre ello tanto los programas gubernamentales de integración como los indígenas en el curso del establecimiento de una educación indígena autodeterminada.

También los Wixaritari, pueblo de la Sierra Madre Occidental, ellos mismos, "desde abajo", y de manera propia, responden al asunto por una educación indígenaautónoma. Teniendo la base en las experiencias concretas de la Comunidad Indígena Autónoma de Bancos de San Hipólito, en ese artículo presentamos el concepto de las capacitaciones y discutimos su papel para un desarrollo autoterminado.

Palabras clave: indigenismo, derechos indígenas, autonomía, capacitaciones.

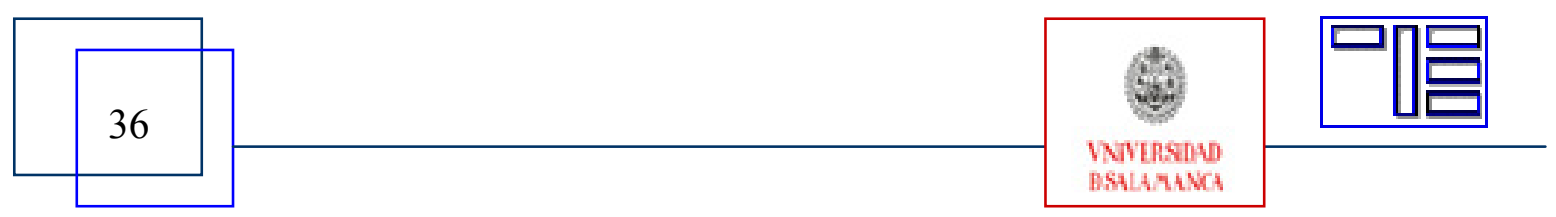




\section{AUTONOMOUS INDIGENOUS FORMATION - ANSWER TO NATIONAL ASIMILATION POLITICS. THE EXAMPLE OF THE WIXARITARI OF MEXICO.}

Abstract. The conditions of the indigenous peoples of Mexico are, inter alia, determined by the national assimilation politics of the Mexican government. Unter the name of the so-called Indigenismo, official programmes for the assimilation and acculturation of the native population are carried out. Rejecting the disregard of indigenous self-reliance and plurality, Mexican indigenous peoples claim the recognition and implementation of their rights and make efforts to put local constructions of autonomy into practice.

However, the sector of education and formation forms an important junction within the debate of indigenous autonomy: it is claimed both by governmental integration programmes and by the indigenas in the course of the establishment of an independent indigenous education.

Also the Wixaritari, people of the Sierra Madre Occidental, respond to the issue for autonomous indigenous formation themselves, "from below", in their own way. In this paper, we present the concept of capacitaciones and discuss its role for a self-contained development on the basis of the concrete experiences of the autonomous indigenous community of Bancos de San Hipólito.

Key words: indigenismo, indigenous rights, autonomy, capacitaciones/formation.

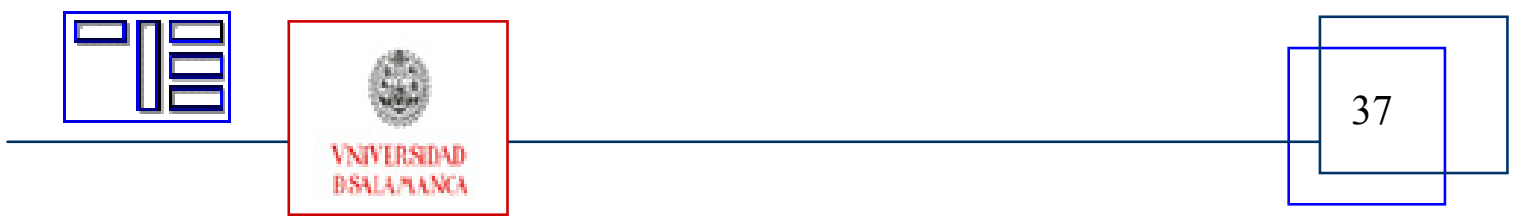


Revista Electrónica Teoría de la Educación.

Educación y Cultura en la Sociedad de la Información.

http://www.usal.es/teoriaeducacion

Vol. 9. No 2. Mayo 2008

\section{FORMATION AUTONOME DES INDIGÈNES - LA RÉPONSE A LA POLITIQUE D'ASSIMILATION: L'EXEMPLE DES WIXARITARI MEXICAINS.}

Sommaire. Les conditions de vie des peuples indigènes mexicains sont définies par une politique d'assimilation du gouvernement Mexicain. Sous le nom de «l'Indigènisme » des programmes officiels sont pratiqués pour assimiler et acculturer les peuples indigènes.

Refusant cet acte comme dédaignage de l'autonomie et diversité, les indigènes réclament l'acceptance et l'implémentation des leurs droits et misent en pratique des formes locales d'autonomie.

Le sujet de l'éducation signifie un focus central dans le discours de l'autonomie indigène :il est considéré par les actions d'intégration officielles du gouvernement, ainsi que par les indigènes développant une éducation originale indigène.

Les Wixaritari, une ethnie de la Sierra Madre Occidental, se donnent la peine de répondre à la question d'une éducation indigène propre, «de la base», de leurs manières. Nous avons l'intention de présenter la conception des capacitaciones à l'exemple des expériences concrètes de la commune autonome indigène de Bancos de San Hipólitos et de discuter l'importance pour un développement indépendant.

Mots- clés: indigenismo, droits indigènes, autonomie, capacitaciones, formation.

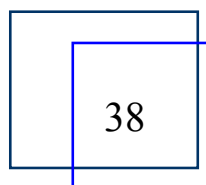




\title{
CAPACITACIONES AUTÓNOMAS INDÍGENAS - RESPUESTA A LA POLÍTICA ASIMILADORA NACIONAL. EL EJEMPLO DE LOS WIXARITAR DE MÉXICO.
}

\author{
Christina Goschenhofer ${ }^{1}$; Santos de la Cruz Carrillo ${ }^{2}$; Carlos Chávez Reyes ${ }^{3}$ \\ 1Christina.Goschenhofer@gmx.de; ${ }^{2}$ ala_cran20@hotmail.com; \\ ${ }^{3}$ rurave 2@yahoo.com.mx \\ Universidad de Tübingen (Alemania). \\ Asociación Jalisciense de Apoyo a Grupos Indígenas, A.C., Guadalajara (México).
}

\section{1.- INTRODUCCIÓN.}

Muchos y múltiples son los esfuerzos para avanzar hacia la autonomía de los pueblos indígenas en América Latina. Cada vez más, la sociedad civil discute estas pretensiones particulares de una autonomía cultural y política. Aparte, aumenta también el interés de la ciencia académica (Gabriel / Latautonomy, 2005). Pero la idea detrás no es nada nuevo para los pueblos indígenas: en un continuo histórico, la resistencia indígena de 500 años se debe considerar como esfuerzo para un status autónomo. La práctica autonómica de los pueblos indígenas es diversa: muchos ejemplos de Nicaragua, Panamá, Ecuador, Bolivia, Brasil y México, entre otros, muestran las diferencias. Las propuestas autónomas no definen ni una ideología ni un régimen o un modelo social (Esteva, 1998). No hay nada más contradictorio para una autonomía que hablar de un modelo, pués cada proceso es único y muestra su desarrollo particular (Gabriel / Latautonomy, 2005).

En México especialmente las propuestas y conceptos de los Zapatistas se considera como emblemáticas de las construcciones de autonomía. Pero el debate de la sociedad civil por formas posibles de autonomía se extiende por más sectores. Por lo tanto, en este texto ponemos el enfoque a las ideas y experiencias con autonomía de los Wixaritari o Huicholes, un pueblo que se encuentra enclavado en los estados de Nayarit, Jalisco, Durango y Zacatecas de la Sierra Madre Occidental de México.

¿Podemos entender autonomía como respuesta, como resistencia, como contrapropuesta al modelo social dominante? ¿Cuáles son las razones, por las que sobre todo comunidades indígenas se enfrentan a la decisión de andar en otros caminos?

Para un análisis parece útil de distinguir dos comprensiones fundamentales de autonomía: autonomía como proyecto político se aplica a la relación entre indígenas y Estado implicando tanto aspectos jurídicos como negociaciones políticas (autonomía de jure). Al otro lado, está la autonomía de facto, la construcción de autonomía en los hechos, la cuál los indígenas realizan en sus comunidades y regiones cotidianamente y

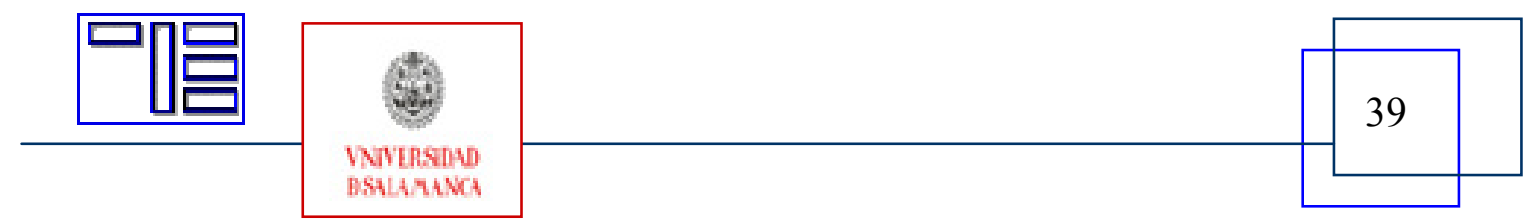


Revista Electrónica Teoría de la Educación.

Educación y Cultura en la Sociedad de la Información.

http://www.usal.es/teoriaeducacion

Vol. 9. No 2. Mayo 2008

la cuál tiene relevancia para problemáticas de desarrollo. Es en este contexto entonces en el que nos interesa preguntar, ¿cuáles son las condiciones y consecuencias de la autonomía que afectan a aspectos de educación y formación?, ¿Cómo se organiza una educación autónoma?, ¿Como son las propuestas de los Wixaritari?

\section{2.- LA SITUACIÓN DE LOS INDÍGENAS EN MÉXICO.}

Los pueblos indígenas crecientemente se vuelven sujetos activos, políticamente independientes en los discursos de la sociedad civil de América Latina. Con sus reivindicaciones por la participación, el reconocimiento de su identidad y de los derechos indígenas, ellos, conscientes de su propia valía, llevan nuevos argumentos a la relación entre el Estado, partes de la sociedad dominantes y los pueblos indígenas. Sin embargo, la situación de los indígenas todavía se encuentra muy lejos de mostrar estructuras igualitarias en el mundo, en Latinoamérica y en México. Siguen siendo parte de los grupos sociales más discriminados y vulnerables (UN / OHCHR, 2001).

Los pueblos indígenas han permanecido expuestos a la práctica por las elites mexicanas del racismo, la discriminación y el paternalismo desde la colonización hasta el presente (Kerkeling, 2003). Actualmente, esa tendencia aún aumenta por la presión de ajuste estructural del encadenamiento económico global, que está obligando la economía nacional de valorizar los territorios indígenas periféricos, privatizar la tenencia comunal y explotar los recursos naturales. La discriminación se manifiesta fuertemente en el llamado indigenismo, corriente ideológica que pretende justificar los intentos estatales de asimilar las formas de vida indígenas con los modos mestizos pues considera a las culturas indígenas como atrasadas respecto a la civilización occidental.

Aunque declaraciones oficiales reconocen la multiculturalidad en la constitución mexicana o en acuerdos internacionales como el Convenio 169 sobre pueblos indígenas y tribales en países independientes de la Organizacón Internacional de Trabajo (OIT), estas expresiones públicas normalmente refieren exclusivamente a las culturas precolombianas - raíz y orgullo de la mexicanidad -. A los indígenas del presente se les sigue tratando con prejuicios: el Estado continua negando su territorialidad y sus gobiernos tradicionales, la vida autóctona actual intenta ser reducida a folklore o residuos atrasados de una forma social anticuada que debe ser "modernizada". El reconocimiento oficial de la pluralidad de otras formas culturales todavía está sobrepuesto por estereotipos peyorativos. El discurso sobre la herencia indígena con frecuencia parece artificial.

La política integracionista no tiende solamente a balancear diferencias culturales dentro de la sociedad mexicana sino también diferencias socioeconómicas. Esa actitud está aportada por la convicción que los pueblos indígenas estarían considerados obstáculo en el camino del adelanto. Según Andrés Aubry, el indigenismo es una respuesta a la pregunta de los mestizos, por qué los países pluriétnicos están atrasados. Con la

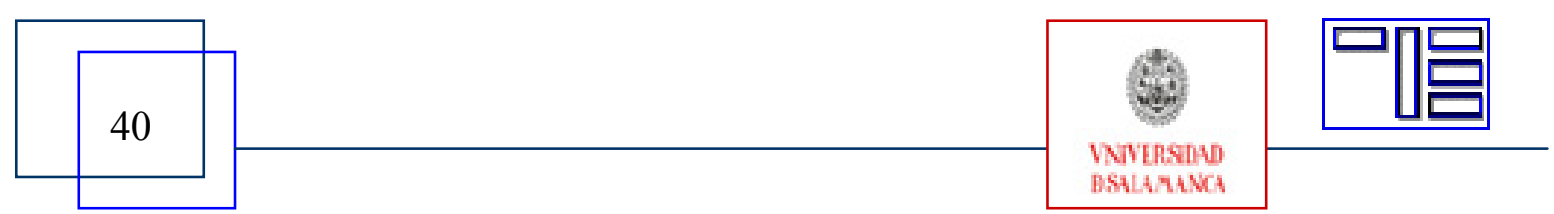


hipótesis que los indígenas sean un freno al desarrollo se desprecia la cultura indígena. Con el hecho que la política indigenista mantiene correspondencia con la necesidad capitalista de consolidar y ampliar el mercado interno y los espacios productivos, el programa del colonialismo interno está realizado a cuenta de los indígenas (Fernández, 2006). Estos directrices modernistas se agravan por las metas neoliberales de la economía, pretensiones de las elites empresarias, aún forzadas por la TLCAN, y de los financiadores internacionales. A través de la planteación de programas gubernamentales dedicados a la "modernización" de las comunidades indígenas, el gobierno cumple con el dictum económico y tiende la inclusión de los indígenas al proyecto del avance nacional.

Con todo, el desconocimiento del derecho a la autodeterminación y la exclusión de procesos de decisión y desarrollo tiene como consecuencia que la población indígena cuenta con la taza más alta de marginación y migración. La pobreza extrema, según criterios del Banco Mundial y la $O N U$, se encuentra sobre todo en municipios indígenas. Son muchos indígenas que son afectados por violaciones múltiples a derechos humanos, por miseria y conflictos territoriales violentos. Esas formas de discriminación y desconocimiento de la autonomía indígena son las circunstancias que marcan la vida cotidiana de la población nativa de México. Son esa discriminación y exclusión, contra las cuáles se forma cada vez más resistencia en todo el país (Kerkeling, 2003).

\section{3.- EL CASO DE LOS WIXARITARI.}

Los Wixaritari son un pueblo indígena en la Sierra Madre Occidental en el oeste de México. Siempre se esforzaron por mantener sus propios formas de vida: hablan activamente el idioma wixárika ; el maíz sigue siendo la base principal de la vida para las familias de los wixaritari y, con eso, fundamento de la organización sociocultural y religiosa de la comunidad. Comunitariamente, reivindican el reconocimiento de sus derechos, defenden el territorio que les legaron sus ancestros contra invasores teiwaris (mestizos) y están intentando de vivir su vida propia.

Junto con otros pueblos indígenas de México, los Wixaritari participan activamente en las reivindicaciones por el reconocimiento de los derechos indígenas y por el discurso de la autonomía. Son uno de los grupos en América Latina, cuál se decidió concientemente para preservar su identidad indígena y sus formas de representación consecuentes y, con eso, hacen quedar al margen otras identidades, al menos temporalmente. Por la concienica aumentanda de la propia valía, los pueblos ponen nuevos retos a la política de minorías del Estado mexicano. Conflictos territoriales, en parte violentos, con comunidades mestizas vecinas son causa y expresión de su problemática fundamental. Los Wixaritari se ven expuestos a múltiples formas de falta de reconocimiento del espacio para su supervivencia y de sus derechos.

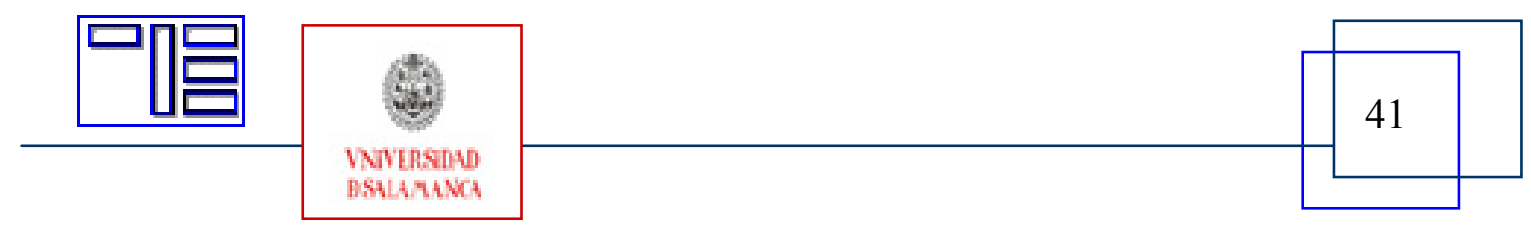


Revista Electrónica Teoría de la Educación.

Educación y Cultura en la Sociedad de la Información.

http://www.usal.es/teoriaeducacion

Vol. 9. No 2. Mayo 2008

Para oponerse a las amenazas a la base territorial de su existencia y a la explotación de sus recursos naturales, una de las comunidades, Bancos de San Hipólito que se encuentra en el Estado de Durango, se declaró autónoma en el año 2002, con la finalidad de impulsar la organización comunitaria y darle vida a la cultura, costumbres y tradiciones, defendiendo a Tatei Yurienaka (la Madre Tierra) a través del agua, el maíz, la medicina tradicional, los lugares sagrados, los bosques, entre otros como esencias vitales del pueblo wixárika.

De esta forma comenzaron a fortalecer espacios de reflexión en los diferentes eventos y ejercer gobierno propio y sistemas tradicionales con una visión integral de la vida y lucha, puntualizando así, paso a paso, una autonomía de facto, frente al exterminio de la clase política dominante y del mismo Estado Mexicano que no ha querido reconocer el territorio ancestral que poseen desde tiempos inmemoriales.

Los wixaritari de Bancos de San Hipólito no cuentan el reconocimiento oficial de sus autoridades autónomas, sino que son una comunidad de hecho, sin embargo, tienen el reconocimiento de las comunidades hermanas de San Sebastián Teponahuxtlán, Santa Catarina Cuexcomatitlán, San Andrés Cohamiata por el Estado de Jalisco, pertenecientes al pueblo wixárika, y la Comunidad de Santa María Ocotán del Pueblo Tepehuano por el Estado de Durango, también el Congreso Nacional Indígena (CNI) como espacio y producto de la suma de muchos esfuerzos y experiencias de los Pueblos Indígenas de México, Grupos Indígenas, Organizaciones de la Sociedad Civil, Universidades, etc. Que en conjunto promueven diversos aspectos del camino que Bancos de San Hipólito ha seguido para lograr sus objetivos de reconocimiento a su derecho a la vida digna y justa. De esta forma, construyendo alianzas es como han tomado la fuerza las autoridades tradicionales y agrarias de Bancos para luchar frente a todo los embates y amenazas que están enfrentando.

Sin embargo, el procedimiento de autonomía de facto todavía es poco común, en comparación con el sistema nacional, y muestra una conciencia muy fuerte de los propios valores sociales y culturales.

\section{4.- EL INDIGENISMO.}

En el curso de la formación del perfil integro y moderno de la república joven de México en los dos siglos pasados, se desarrolló la política del indigenismo. Con base en el pasado indígena, la herencia española y el presente mestizo, se dio atención extraordinaria a la creación de una identidad nacional que abarcara a todos los mexicanos. En ese sentido, el indigenismo tuvo su función en ser instrumento para deshacer las identidades culturales étnicas indígenas, asimilarlas, homogenizarlas, occidentalizarlas y integrarlas en el concepto del mestizaje para lograr el nation building.

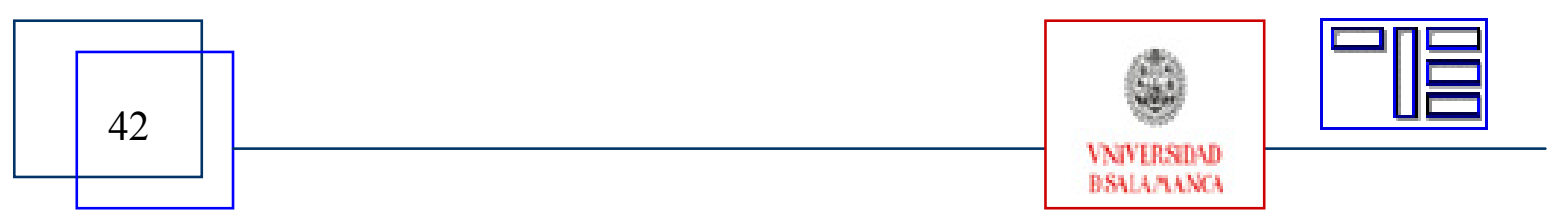


La realización de la transformación cultural dirigida llegó a una primera cima con la fundación del Instituto Nacional Indigenista (INI) en 1948 (a partir de 2003 renombrado en Comisión Nacional para el Desarrollo de los Pueblos Indígenas, CDI). En esa planeación de integración y desarrollo de los pueblos indígenas funcionalizada por el Estado, se puso atención especial a educación, idioma y cultura (CDI, 2006). A través de concebir programas institucionales de desarrollo para las comunidades indígenas, especialmente en los áreas de educación, salud, nutrición y agricultura, el Estado pretende la inclusión de los indígenas en el proyecto del desarrollo nacional, impuesto desde arriba.

Por parte de muchas comunidades, se trata a los funcionarios gubernamentales con desconfianza, después de decenas de experiencia tanto con fracasos del desarrollo y explotación de saberes tradicionales como con la tendencia de fijar las culturas indígenas como folklore y usarla para objetivos comerciales ("Etnoturismo"). Además, acusan al INI / CDI que hasta el día no han sido capaces de aliviar la miseria ni la discriminación económica y social. Más fuerte expresan su crítica al indigenismo los antropólogos A. Barabás y M. Bartolomé, miembros del International Work Group for Indigenous Affairs de la ONU, cuando publicaron en 1973 por primera vez a nivel internacional, la denuncia de la existencia de un programa de etnocidio, que se aplica en México como elemento del desarrollo capitalista por parte del gobierno a través de instituciones del desarrollo regional con las minorías étnicas (citados en Mayer, 1998).

\section{5.- POLÍTICA INDIGENISTA DE EDUCACIÓN.}

Las instituciones de educación tienen gran importancia por su influencia a partir de la niñez. Aunque, las escuelas bilingües tienen una gran extensión, el problema es muy sutil: los valores y los contenidos de la «cultura nacional», transmitidos en las escuelas, forman una contradicción al modo a vivir indígena. Junto con la larga ausencia de los niños de sus familias, cuándo están en los albergues durante la semana, eso puede conducir a una alienación profunda o a un conflicto de identidades para los niños y jóvenes. La organización de la currícula es diseñado igual para todo el país; casi no se orienta a las necesidades y intereses de los pueblos indígenas distintos.

En la Sierra Wixárika están 35 escuela primarias y algunas telesecundarias, luego de este nivel la continuación de la formación para los jóvenes solo es posible en las ciudades, esto implica gran carga económica para las familias, una vida bajo condiciones alienantes y el riesgo de ser desenraizados de su propia cultura. Con eso, los jóvenes están enfrentados a un reto enorme a su identidad.

Del reporte del Centro Coordinador para el Desarrollo de la Región Huicot del año 1975 (Palafox Vergas) resulta el no-reconocimiento de la cultura indígena:

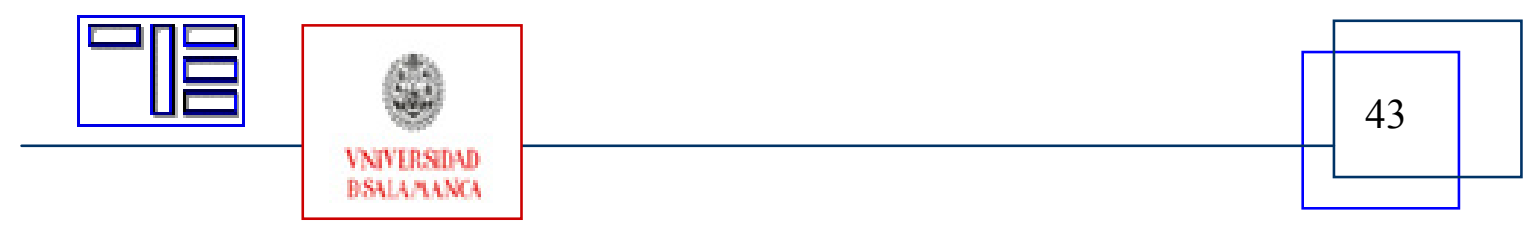


Revista Electrónica Teoría de la Educación.

Educación y Cultura en la Sociedad de la Información.

http://www.usal.es/teoriaeducacion

Vol. 9. No 2. Mayo 2008

Siempre se pensó en que la educación constituye un gran vehículo para integrar a las comunidades indígenas, al patrón de cultura del pueblo mexicano e incorporarla a las actividades propias del resto de la población del país. (....) La economía de autoconsumo y la cultura tradicional a que ha estado sujeto el niño indígena, fue una de las tareas a vencer por los primeros maestros y promotores para que los padres de familia permitieran a sus hijos asistir a la escuela.

La responsabilidad para la educación del niño indígena ha sido retirada de las familias y comunidades y transferida a las manos estatales.

Mientras, Artículo 27 del Convenio 169 de la OIT en su parte de Educación y Medios de Comunicación define las siguientes pautas para una política de educación adecuada para grupos indígenas, debiendo indicarse que el convenio es vigente en el país desde $1990 \mathrm{y}$, por lo tanto, es obligatorio para el Estado Mexicano ante el derecho internacional:

1. Los programas y los servicios de educación destinados a los pueblos interesados deberán desarrollarse y aplicarse en cooperación con éstos a fin de responder a sus necesidades particulares, y deberán abarcar su historia, sus conocimentos y técnicas, sus sistemas de valores y todas sus demás aspiraciones sociales, económicas y culturales.

2. La autoridad competente deberá asegurar la formación de miembros de estos pueblos y su participación en la formulación y ejecución de programas de educación, con miras a transferir progresivamente a dichos pueblos la responsabilidad de la realización de esos programas, cuando haya lugar.

3. Además, los gobiernos deberán reconocer el derecho de esos pueblos a crear sus propias instituciones y medios de educación, siempre que tales instituciones satisfagan las normas mínimas establecidas por la autoridad competente en consulta con esos pueblos. Deberán facilitárseles recursos apropiados con tal fin.

\section{6. - LA IMPLEMENTACIÓN DE LOS DERECHOS INDÍGENAS EN MÉXICO.}

El choque de los intereses culturales y económicos distintos, o sea, de las elites no indígenas y los grupos indígenas esforzándose por conseguir su autonomía, tiene por consecuencia la irrupción de conflictos que el estado no es capaz a resolver. Al contrario, por legislación y decisiones jurisdiccionales está promoviendo las pretensiones de los actores mestizos, mientras sigue la discriminación a los indígenas y la negación de sus reivindicaciones. En los conflictos, se violenta sistemáticamente tanto los derechos humanos como los derechos indígenas oficialmente reconocidos.

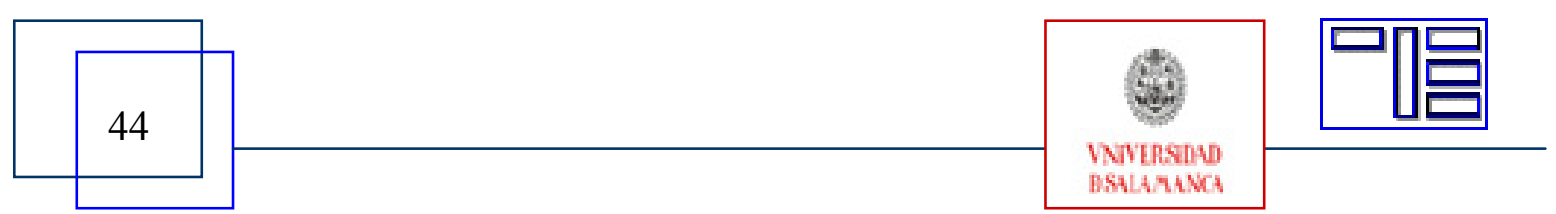


Instituciones internacionales de defensoría de los derechos humanos expresan su preocupación.

Como respuesta a las amenazas a su modo de vivir, se ofrecen dos formas de autodefensa a los pueblos indígenas de México. Por un lado, pueden usar la estrategia de provocar una reacción del Estado reconocer y llevar a la práctica los derechos a través de vías jurídicas y participando en el discurso político. Acuerdos internacionales pueden ser utilizados como instrumentos, autoridades correspondientes de la ONU, OIT o de la Corte Interamericana de Derechos Humanos pueden ser llamados.

Con la aplicación directa del Convenio 169 de la OIT por parte de la comunidad de Bancos de San Hipólito a través de una queja presentada por la Unión de Comunidades Indígenas Huicholas de Jalisco (UCIHJ), lo cual dió lugar a una recomendación favorable a los indígenas en 1998 (la primera en el mundo) del Consejo de Administración de la OIT, que resolvió la demanda territorial pidiendo al gobierno mexicano resolver las causas que dieron lugar a la reclamación. Posteriormente se emitió un reporte especial en el año de 2006, en que la Comisión de Expertos para la Aplicación de Convenios y Recomendaciones (CEACR) de la OIT, por el nulo avance del gobierno mexicano en el cumplimiento de la recomendación de 1998, y le solicita que incluya el caso de Bancos entre los prioritarios al nivel nacional para su solución.

Aislados del gobierno mexicano, los Wixariari mostraron su conocimiento de la importancia de las instituciones internacionales en el debate por los derechos indígenas y en la conversión de los derechos indígenas a la práctica en el marco nacional, es decir, a través de legislación, jurisdicción y planteamiento de programas estatales, sin embargo, continuamente del Estado Mexicano da pruebas de su impotencia para implementarles.

\section{7.- LA OPOSICIÓN : IDEAS INDÍGENAS DE AUTONOMÍA.}

Desde la perspectiva indígena sí se ve de gran valor los intentos jurídicos. Sin embargo, a causa de los procesos jurídicos lentos y la desilusión frente la política actual, están llamados a mejorar autónoma y rápidamente su situación. Por eso, muchas comunidades de la población autóctona mexicana ya realizan y construyen la autonomía en los hechos en diferentes niveles, mientras tanto, el proceso crece en significados (Esteva, 1998).

La idea de la autonomía tiene la meta de equipar los afectados con más poder, es decir, reconocer a los pueblos indígenas y otros grupos sociales discriminados más control sobre su espacio de vida y dar opciones de decidir en lugar de seguir determinados por la pobreza, opresión y marginación. Gustavo Esteva considera la autonomía como forma de democracia radical, como democracia "desde abajo", como poder del pueblo. Con eso entiende la capacidad de la gente de gobernarse a si mismos y de ejecutar poder a través sus autoridades. Ellos, solamente deban actuar según el principio tradicional de

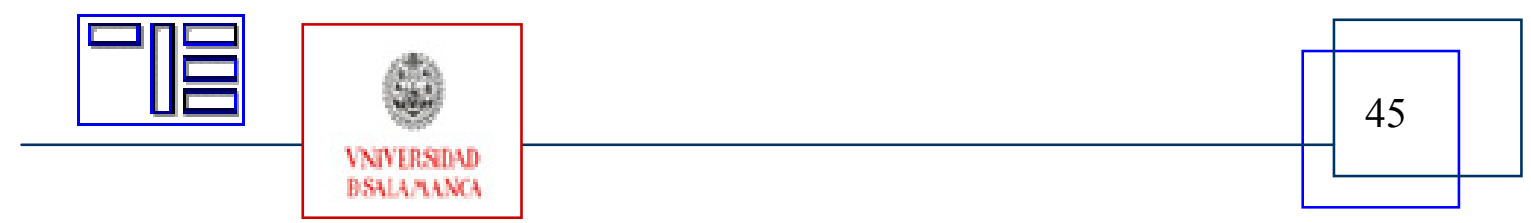


Revista Electrónica Teoría de la Educación.

Educación y Cultura en la Sociedad de la Información.

http://www.usal.es/teoriaeducacion

Vol. 9. No 2. Mayo 2008

«mandar obedeciendo». Por eso, ese entendimiento conceptual es una propuesta para una transformación profunda, abarcando a toda la sociedad, abierta para todos. Transformación que fortalecería la integridad del Estado justamente por esa forma de democracia del pueblo (Esteva, 1998).

Las formas de autonomía, democracia viva y resistencia son múltiples - también en México: "Zapatismo did not, invent' the indigenous struggle, but gave it a national dimension, stimulated ist growth, [and] unified many of ist currents." (Hernández Navarro, 1998, 26, citado en Levi, 2002, 30). Con consciencia creciente como actores sociopolíticos, los indígenas mexicanos expresivamente usan su identidad como instrumento político - tanto de manera simbólica como estratégica -. El Congreso Nacional Indígena (CNI) es considerado uno de los importantes vínculos estructurales de la red de la oposición indígena.

Cornelius Castoriadis conecta, en su entendimiento, la idea de la autonomía colectiva con la palabra y subraya el papel de la discusión de la asamblea. La asamblea no sólo forma la institución comunitaria legislativa sino también crea otras instituciones; continuamente las critica y cambia en lugar de ser determinada por ellas (Chameau, 2005). El principio de las comunidades locales de la asamblea, orientada a la reflexión y discusión colectiva, y, adaptando ese principio, de los encuentros de la red de la sociedad civil indígena pueden ser entendidos correspondientemente.

La autonomía en los hechos significa para muchos indígenas mexicanos la herramienta para un desarrollo autodeterminado. La decisión de adherirse a la conversión a la práctica de la autonomía y transponerla como expresión política en el discurso de la sociedad civil, tiene correlación con el grito "Ya basta!". Lleva un contenido explícitamente emancipador que afecta a la consciencia colectiva: los indígenas conocen su capacidad de autogobernarse:"Nosotros llevamos a cabo las iniciativas" (Gutiérrez y Palomo, 1999, 79).

"En muchas regiones del país los pueblos ejercen ya su autonomía en los hechos. Hacemos reuniones, talleres, encuentros, pero también fortalecemos nuestras asambleas, nuestras autoridades agrarias y tradicionales, la lucha en defensa de nuestros maices, la defensa de nuestros bosques y agua, la lucha contra la certificación de nuestras tierras y los servicios ambientales, ejerciendo una educación cada vez más autónoma."

El parágrafo, citado por Vera Herrera (2006) de la declaración del 4o encuentro del CNI en 2006 reúne elementos fundamentales de la organización interna para el proceso de autonomía. Dichos puntos expresan un entendimiento integral de la autonomía viva, autonomía situada en el espacio de la vida cotidiana directamente (Gutiérrez y Palomo, 1999, 83). La comunidad local y su organización son punto de referencia y de salida de un desarrollo autodeterminado. Aspectos de educación, formación y capacitación automáticamente están incluidos.

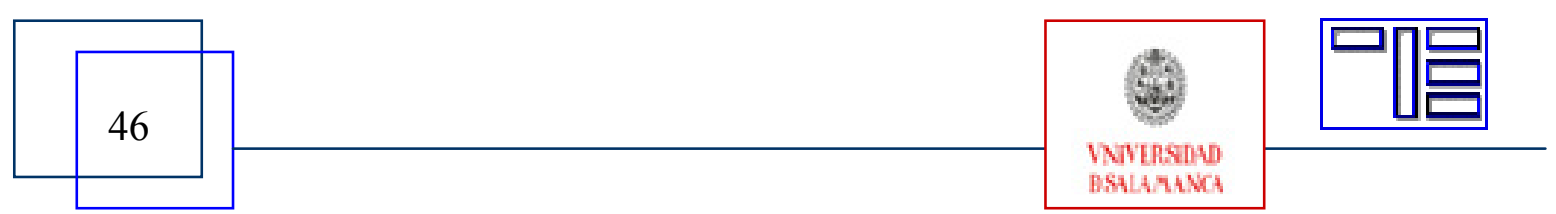




\section{8.- AUTONOMÍA EN LOS HECHOS - EL EJEMPLO WIXÁRIKA DE CAPACITACIÓN.}

La autonomía los indígenas mexicanos entienden como proceso dinámico hacia una autodeterminación cada vez más integral. Con la autonomía, las comunidades wixárikas forman las circunstancias de la vida, así en responsabilidad propia para el futuro de la comunidad. Sólo por la autonomía les da la posibilidad de vivir su modo de vida con la amplitud que ellos quieren. La capacitación es una herramienta importante para ampliar y consolidar la autonomía. En este artículo ponemos el enfoque sólo en la educación informal de las capacitaciones sin reconocer los intentos curriculares en un contexto escolar, que también tienen lugar en la Sierra Wixárika.

Los Wixaritari forman parte activa de la red indígena nacional: favorecen alianzas con otros pueblos y grupos indígenas como la cooperación con sus pueblos vecinos Tepehuanos y Coras, y atienden los encuentros del CNI. A nivel nacional, a veces también continental, participan en encuentros y talleres de información e intercambio, por ejemplo en los temas de territorio y protección del medio ambiente, servicios ambientales, geopolítica, defensa del maíz y del agua, medicina tradicional y agricultura sustentable. De las experiencias de la lucha por títulos territoriales, los Wixaritari conocen la fuerza de alianzas, son estas alianzas, foros y talleres, que tejen una red indígena mexicana de intercambio y solidaridad. Esa red de información puede ser considerada como una instancia de capacitación a nivel supralocal.

Al nivel local, la autonomía implica primeramente la organización interna de la comunidad: el conocimiento y la salvaguardia del costumbre, o sea, de la propia historia y los mitos, la participación en las ceremonias y el respeto de las decisiones de las autoridades tradicionales y agrarias así como de asamblea. Ese fortalecimiento de lo propio está complementado por la apertura hacia nuevas ideas, en ese sentido, talleres y asambleas estaban introducidos para evaluar el status quo, hacer diagnósticos de los problemas locales y plantear planeaciones de acciones a futuro. Las memorias de estos encuentros documentan tanto la forma en que estos dieron inicio a un proceso colectivo de toma de consciencia como la perspectiva amplia de las decisiones. La planeación de estrategias de desarrollo local concede gran importancia a los talleres en el contexto de la autonomía. Una de las metas es la integración de educación, salud, cultura y política en los ciclos de trabajo durante las planeaciones.

En favor de una distribución efectiva de trabajos, se formaron comités comunitarios para distintos aspectos de desarrollo y áreas de trabajo. En las comunidades del pueblo wixárika hay grupos para la atención en áreas de economía, medio ambiente, análisis territorial, almacenes comunitarios, panadería y transporte. Los integrantes de los comités estan nombrados por la asamblea general. El mandato dispone a los encargados para actuar ligadamente a los acuerdos colectivos. En talleres están capacitados, se discute ideas e intercambian las experiencias de los Comités de cada comunidad.

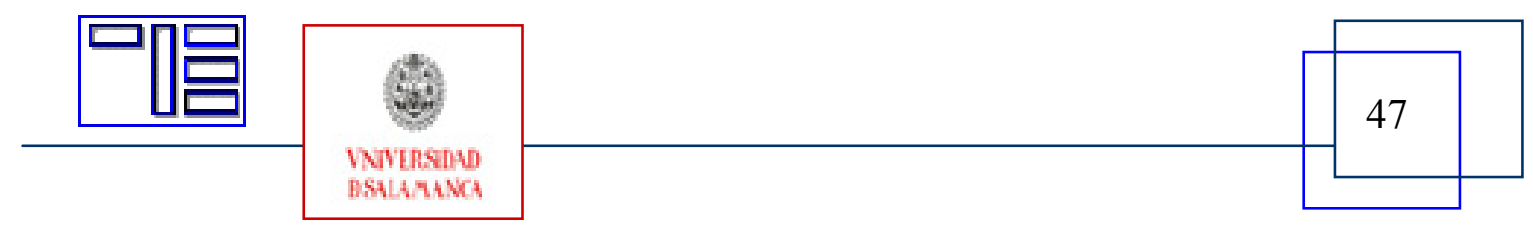


Revista Electrónica Teoría de la Educación.

Educación y Cultura en la Sociedad de la Información.

http://www.usal.es/teoriaeducacion

Vol. 9. No 2. Mayo 2008

En el caso de Bancos de San Hipólito la comunidad formó los siguientes comités comunitarios por medio de los talleres de capacitación y planeaciones que ha desarrollado:

1. Comité del Almacén. Con la idea de formar grupos capacitados en contabilidad y administración que respondan a la comunidad y al fortalecimiento de la economía solidaria.

2. Comité de Economía. Se creó con la finalidad de vigilar los trabajos y proyectos que ha ejercido la comunidad. Operando a base de planeaciones y evaluaciones directamente con los miembros de los proyectos.

3. Comité de Ecología. Surge con la conciencia de conservar y defender el medio ambiente, el agua, los manantiales, el suelo, los lugares sagrados y todo lo que existe en la madre tierra. Ejerciendo las prácticas de agricultura orgánica y haciendo una serie de investigaciones sobre temas de maíz, bosques, recuperación de saberes locales y tradicionales.

4. Grupo de Análisis Territorial. Surge por la necesidad de medir el territorio para la solución del conflicto agrario, organizándose y capacitándose con los miembros de otras comunidades. Ahora han pasado de las mediciones agrarias a planteamientos geográficos para los ordenamientos comunitarios. Se considera una organización comunitaria y regional importante para la defensa territorial.

De esta forma se viene fortaleciendo y consolidando la autonomía regional como el camino más práctico de organización y de toma de decisiones para acciones más enérgicas frente al Estado Mexicano.

Un significado primario se da en la formación y la carrera académica de jóvenes, para que transfieran sus saberes como multiplicadores para el bien de las comunidades. A los Wixaritari, que están estudiando en Guadalajara, la Unión de Jóvenes Estudiantes Wixaritari (UJEW) les da estabilidad y respaldo mutuo para enfrentarse a los retos de la vida urbana. Un proyecto escolar en la Sierra Wixárika, existente desde 1995, está estableciendo una secundaria con propio currículo reconocido por la Secretaría de Educación Pública.

Construyendo la autonomía viva la reflexión y el revivir de saberes tradicionales y locales, así como la domesticación de nuevas problemáticas y técnicas se volvieron tareas cotidianas. Con eso, la comunidad forma la plataforma para un proceso colectivo de aprendizaje. El principio de la capacitación se vuelve herramienta para el establecimiento de la autonomía. Así, recuerda al concepto de la concientización de Paulo Freire: A través un proceso crítico y reflexivo, los comuneros deben concientizar su opresión, justamente en sentido cultural, y su mentalidad colonizada y asimilada a la corriente de pensamiento hegemónica para, por fin, recordar a su propia cultura y

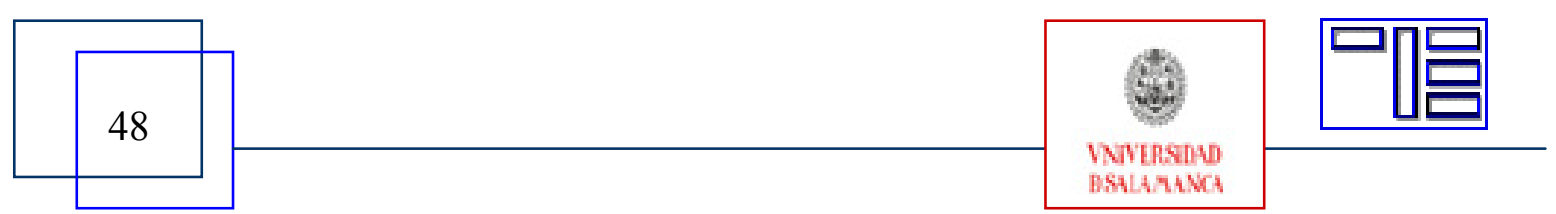


identidad (Johnson; Louis; Pramono, 2006). La construcción de la autonomía en los hechos da espacio a un proceso de reflexión, que, como una reacción en cadena, fortalece la consciencia de responsabilidad colectiva de la comunidad. Son ese espíritu comunitario y el intercambio regional, los que organizan el desarrollo en el territorio wixárika.

\section{9.- CONCLUSIONES.}

La oposición a la política de asimilación, promovido sobre todo por el sector nacional de educación, está aumentando con el debate por los derechos indígenas. Los múltiples ejemplos de la autonomía en los hechos de las comunidades da testimonio de esos sucesos. Por eso, está puesta en marcha una dinámica creciente en el discurso entre Estado, sociedad dominante y pueblos indígenas.

Los indígenas participan en esa discusión con conciencia creciente de la propia valía, a través de la cooperación (inter)nacional y de reivindicaciones más precisas, pero también con el fortalecimiento continuo de las estructuras propias. Las iniciativas locales se enlazaron con la red nacional de la sociedad civil, que da importancia a la comunidad como piedra angular para un proceso de aprendizaje colectiva.

Mientras todo, se debe entender la autonomía en los hechos como un concepto más allá de normas, un concepto siempre cambiando y en construcción. Correspondientemente, es un proyecto abierto, colectivo, en proceso continuo, constituido por la reflexión comunitaria de orientaciones, instituciones y prácticas, avanzando en su realización en el camino hacia la utopía (el futuro), pero al mismo tiempo, siempre debe haber existido (el pasado y presente permanente). Es más anárquico como para que pueda estar normado o fijado, y más integral como para que las leyes puedan captarlo.

Los procesos múltiples de las construcciones de autonomía locales y regionales son objetos complejos e híbridos, compuestos por un lado, por tradiciones e identidad histórica y por otro lado, por alianzas actuales, políticamente razonadas y con la adopción de nuevos conceptos. Por lo tanto debe ser obvio de reconocer el potencial creativo y el efecto movilizador, cohesivo y emancipador del proyecto de la autonomía comunitaria. Correspondientemente, también las capacitaciones autónomas son parte del proceso hacia la autonomía, son múltiples y creativas y sobre todo autodeterminadas. Esa educación autónoma es un instrumento en el camino hacia un desarrollo propio y autodeterminado.

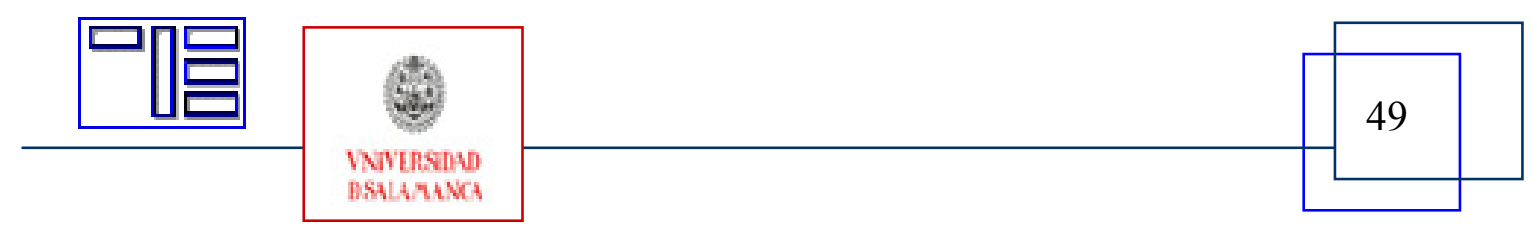




\section{0.-BIBLIOGRAFÍA.}

COMISIÓN NACIONAL PARA EL DESARROLLO DE LOS PUEBLOS INDİGENAS (2006). Documentos Generales. Consultado el 01 de febrero de 2008 en: http://cdi.gob.mx/index.php

COMISIÓN DE EXPERTOS EN APLICACIÓN DE CONVENIOS Y RECOMENDACIONES (2006). Observación individual sobre el Convenio sobre pueblos indigenas y tribales (No. 169). Ginebra. ILO.

CHAMEAU, M. (2005): Das Konzept der Autonomie bei Ivan Illich und bei Cornelius Castoriadis, en Archipel $\mathrm{n}^{\mathrm{o}}$ 131,10/05. $<\mathrm{http}: / / \mathrm{www}$.civicforum.org/index.php?lang=DE\&site=ARCHIPEL\&sub_a=ARCHI_131\&article $=735>$ [Consulta: 20/10/06].

ESTEVA, G. (1998). Autonomía y democracia radical: el tránsito de la tolerancia a la Hospitalidad. En BARTOLOMÉ, M. A. y BARABAS, A. M. (Coord.): Autonomías étnicas y Estados nacionales, (pp. 307 - 332). México: Instituto Nacional de Antropología e Historia y Consejo Nacional para la Cultura y las Artes.

FERNÁNDEZ, J. M. (2006). Indigenismo, en Diccionario Crítico de Ciencias Sociales. Universidad de Madrid. http://www.ucm.es/info/eurotheo/diccionario/I/indigenismo.htm [Consulta: $5 / 11 / 06]$.

GABRIEL, L. y LATAUTONOMY (Coord.) (2005). Politik der Eigenständigkeit. Lateinamerikanische Vorschläge für eine neue Demokratie. Viena: Mandelbaum.

GUTTIÉRREZ, M. y PALOMO N. (1999). Autonomía con Mirada de Mujer. En BURGUETE CAL y MAYOR, A. (Coord.): México: Experiencias de Autonomía Indígena, (pp. 54 - 86). Copenhague: IWGIA.

INTERNATIONAL LABOUR ORGANIZATION (2003). Convenio Número 169 sobre Pueblos Indígenas y Tribales. Un manual. Ginebra: ILO

JOHNSON, J. T. ; LOUIS, R.P. y HADI PRAMONO, A. (2006). Facing the Future: Encouraging Critical Cartographic Literacies in Indigenous Communities. En ACME: An International E-Journal for Critical Geographies, (pp. 80-98). Consultado el 19 de marzo de 2006 en: http://www.acme-journal.org

KASTNER, J. (2006). Wille zur Freiheit. Autonomie in der entwicklungspolitischen Diskussion, en Iz3W 294, (4), 16 - 19.
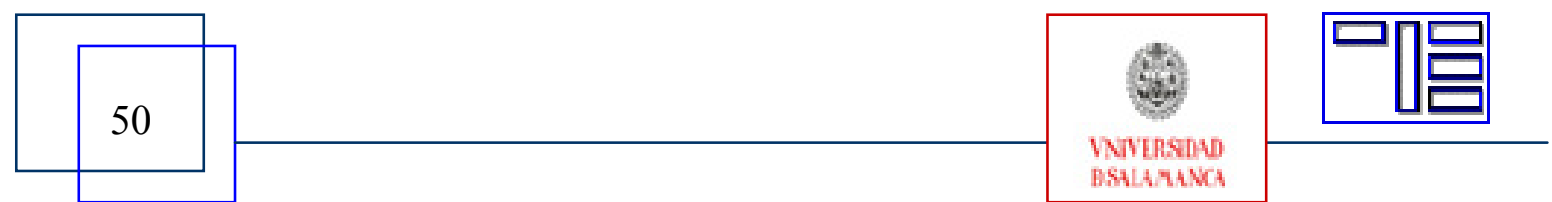
KERKELING, L. (2003). La Lucha Sigue! Der Kampf geht weiter! EZLN - Ursachen und Entwicklungen des zapatistischen Aufstands. Münster: Unrast - Verlag.

LEVI, J. M.(2002). A New Dawn or a Cycle Restored? En MAYBURY-LEWIS, D. (Coord.). The Politics of Ethnicity: Indigenous Peoples in Latinamerican States. Harvard: Harvard Press.

MAYER, G. (1999). Interner Kolonialismus und Ethnozid in der Sierra Tarahumara (Chihuahua, Mexiko). Bedingungen und Folgen der wirtschaftsräumlichen Inkorporation und Modernisierung eines indigenen Siedlungsraumes. Tübingen: Tübinger Beiträge zur geographischen Lateinamerika-Forschung (TBGL).

PALAFOX VERGAS, M. (1975). El Plan Huicot. Obra humanista de la revolución. México: Tepic.

STAVENHAGEN, R. (1997). Indigene Völker: Neue Akteure in Lateinamerika. En GLEICH, U. (coord.): Indigene Völker in Lateinamerika: Konfliktfaktor oder Entwicklungspotenzial?, (pp. 15 - 33). Frankfurt am Main: Vervuert Verlag

UN / OHCHR (2001). United Nations Guide for Indigenous Peoples. Geneva. $<$ http://www.unhchr.ch/html/racism/00-indigenousguide.html $>$ $15 / 11 / 06]$.

[Consulta:

VERA HERRERA, R. (2006). Congreso Nacional Indígena: Un espacio de lucha en la Otra Campaña, en Ojarasca $\mathrm{n}^{\mathrm{o}} 111, \quad 7 / 2006$. $<$ http://www.jornada.unam.mx/2006/07/15/oja111.html> [Consulta: 5/12/06].

Para citar este artículo puede utilizar la siguiente referencia:

GOSCHENHOFER, Christina, DE LA CRUZ CARRILLO, Santos \& CHÁVEZ REYES, Carlos (2008). Capacitaciones autónomas indígenas. Respuesta a la política asimiladora nacional. El ejemplo de los Wixatar de México. En APARICIO, Pablo (Coord.) Desde la diversidad hacia la desigualdad: ¿destino inexorable de la globalización? [monográfico en línea]. Revista Electrónica Teoría de la Educación: Educación y Cultura en la Sociedad de la Información. Vol. 9, nº 2. Universidad de Salamanca. [Fecha de consulta: dd/mm/aaaa]. $<$ http://www.usal.es/ teoriaeducacion/rev_numero_09_02/n9_02_goschenhofer.pdf $>$ ISSN 1138-9737

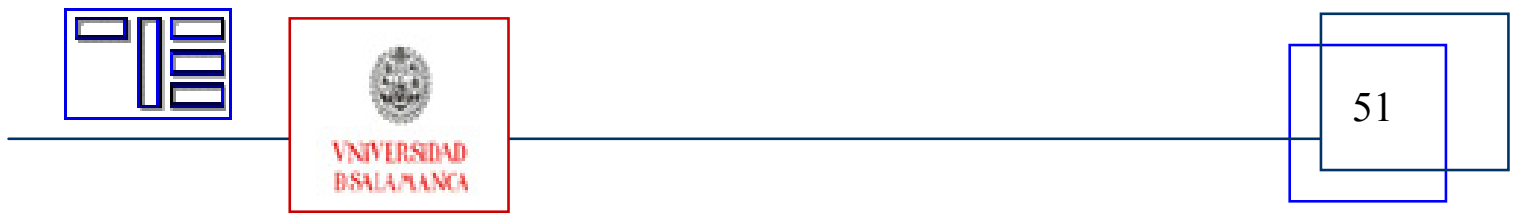

